\title{
Herschel-Bulkley Magnetized Blood Flow Model for an Inclined Tapered Artery for an Accelerated Body
}

\author{
Neetu Srivastava* \\ "Department of Mathematics, Amrita School of Engineering, Bengaluru, Amrita Vishwa \\ Vidyapeetham, India, 560035.
}

Received 26 December 2017; accepted 27 June 2018; available online 30 June 2018

DOI: https://10.30880/jst.2018.10.01.008

\begin{abstract}
Analytical investigation of Herschel-Bulkley model for axisymmetric pulsatile blood flow through an inclined stenosed artery of a periodically accelerated body under the influence of a magnetic field has been done. Invoking suitable transformations, the flow governing partial differential equations are non-dimensionalized. For these non-dimensionalized equations, an exact solution representing the different flow characteristic has been derived by employing the perturbation method. Flow rate and impedance analysis of the Herschel-Bulkley fluid has been done graphically by varying the yield stress, pressure gradient. Some important results are obtained pertaining to the medical interest.
\end{abstract}

Keyword: Herschel Bulkley fluid; Blood flow; Inclined tapered artery.

\section{Introduction}

Human body tolerance to acceleration (due to travelling etc.) depends on some of the vital facts including the duration of acceleration stress. Mathematical analysis has a significance to quantify the effects of periodic acceleration in an inclined stenosed artery. These problems can be used to describe the effect of body accelerations on the physiologically important flow dynamics of human blood flow through artery. The above mentioned fact has exhilarated the study of flow through constrictions during the past decade. Realizing the fact that pulsatile nature of the flow cannot be neglected, many theoretical analysis and experimental studies of the flow through stenosis have been performed.

Abdullah et.al. [1] studied the micropolar model for axi-symmetric blood flow through an axially non-symmetric but radially symmetric mild stenosis tapered artery. Abdullah et.al. [4] investigated numerically the magnetohydrodynamics (MHD) effects on the blood flow. It was found that an applied magnetic field reduces the blood flow rate. Bodnar et.al. [5]described and discussed the results of numerical comparative study performed in order to demonstrate and quantify some of the most relevant nonNewtonian characteristics of blood flow in medium-sized blood vessels, namely, its shearthinning and viscoelastic behavior. Mekheimer and Elkot [6] investigated the influence of heat and chemical reactions on blood flow through anisotropically tapered elastic artery with time-variant overlapping stenosis. The nature of blood in small arteries has been analyzed mathematically by considering it as a Sisko fluid. Ali et.al. [7] presented a mathematical study for unsteady pulsatile flow of blood through a tapered stenotic artery using a finite difference method. Akbar and Nadeem [8] examined the blood flow through tapered artery with stenosis. The non-Newtonian nature of blood in small arteries has been analyzed mathematically by considering the blood as Carreau fluid. Srivastava [2] has investigated analytically blood flow in a porous inclined stenotic artery under the influence of the inclined magnetic field. Srivastava [3] has investigated the Casson model for axisymmetric pulsatile blood flow through an inclined stenosed artery of a periodically accelerated body under the influence of a magnetic field.

Priyadharshini et.al. [9] has analysed that the blood flow through a tapered artery with stenosis and dilatation has been carried out where the blood is treated as incompressible Herschel-Bulkley fluid. A comparison between numerical values and analytical values of pressure gradient at the midpoint of 
stenotic region shows that the analytical expressions for pressure gradient works well for the values of yield stress till 2.4. Sriyab [10] has derived the expressions for velocity, plug flow velocity, wall shear stress, and the flux flow rate for the Herschel Bulkley fluid model. Singh [11] developed a mathematical model for studying the magnetic field effect on blood flow through an axially non-symmetric but radially symmetric atherosclerotic artery. Herschel-Bulkley equation has been taken to represent the non-Newtonian character of blood. The response of magnetic field, stenosis height, shape parameter on velocity, volumetric flow rate in stenotic section and wall shear stress at the surface of stenosis are revealed analytically and graphically

With this motivation and purpose, a mathematical model for Herschel-Bulkley fluid through stenosed inclined artery of an accelerated body in the presence of an inclined magnetic field has been considered. In this paper, an attempt has been made to derive and study the physiologically important flow quantities such as volumetric flow rate, impedance for blood flow in an artery by modeling blood as Herschel-Bulkley fluid. The physical quantities involved in the problem are non-dimensionalized and the expressions for flow quantities such as velocity, flow-rate, wall shear stress and longitudinal impedance of the artery are obtained for pulsatile flow. Assuming that the stenoses are mild, analytical solutions have been obtained, using the perturbation method.

\section{Mathematical Formulation}

Consider an axially symmetric, laminar, pulsatile, and fully developed flow of blood (assumed to be incompressible) in the axial direction through a circular narrow artery with constriction. The constriction in the artery is assumed as due to the formation of stenosis in the lumen of the artery and is considered as mild. In this study, we consider the shape of the stenosis, as asymmetric. The geometry of segment of a narrow artery with asymmetric shape of mild stenosis is shown in Fig. 1(a). The segment of the artery under study has been considered to be long enough so that the entrance, end, and special wall effects can be neglected. Due to the presence of the stenosis in the lumen of the segment of the artery, it is appropriate to treat the segment of the stenosed artery under study as rigid walled. Assume that there is periodical body acceleration in the region of blood flow and blood is modelled as non-Newtonian fluid model with yield stress. In this study, we use Herschel-Bulkley non-Newtonian fluid models with yield stress. It has to be noted that Herschel-Bulkley fluid model's constitutive equation reduces to the constitutive equation of Newtonian fluid when the yield stress parameter becomes zero. The cylindrical polar coordinate system $(r, \theta, z)$ has been used to analyze the blood flow.

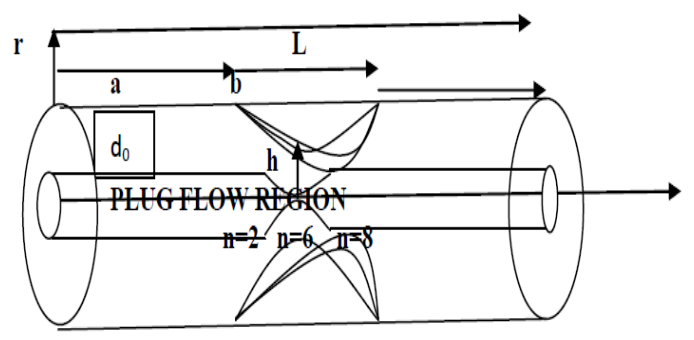

(a)

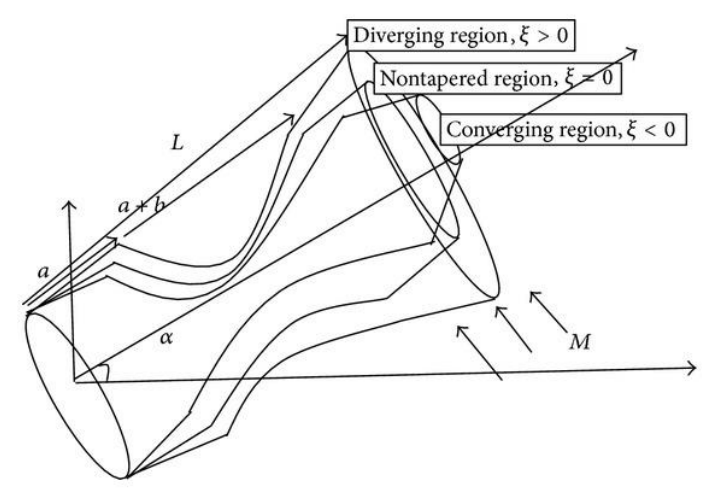

(b)

Fig.1 (a) Geometry of a stenosed artery and (b) Schematic of tapered artery region.

\section{Governing Equation and Boundary Condition}

The momentum equations governing the blood flow in the radial and axial directions can be written as:

$$
\begin{aligned}
& \overline{\rho_{H}}\left(\frac{\partial \overline{u_{H}}}{\partial t}+g \sin \alpha\right)=-\frac{\partial \bar{p}}{\partial \bar{z}}-\frac{1}{\bar{r}} \frac{\partial\left(\bar{r} \tau_{H}\right)}{\partial \bar{r}}+ \\
& \bar{F}(t)+\mu_{0} M \frac{\partial \bar{H}}{\partial \bar{z}} \cos \psi, \\
& \frac{\partial \bar{p}}{\partial \bar{r}}=0
\end{aligned}
$$


where $\bar{r}$ and $\bar{z}$ denotes the radial and axial coordinates respectively, is magnetic permeability, $\mathrm{M}$ is magnetisation of fluid particles, $\bar{H}$ is magnetic field intensity, $\alpha$ is the inclination of artery, $\psi$ is the angle of inclined magnetic field, $\bar{P}$ is the pressure, $\bar{t}$ is the time, $\overline{\tau_{H}}$ is the shear stress of the Herschel Bulkley fluid, $\bar{F}(t)$ is the term which represents the body acceleration and is given by,

$$
\bar{F}(t)=\overline{a_{0}} \cos \left(\omega_{b} t+\phi\right)
$$

where $\overline{a_{0}}$ is the amplitude of the body acceleration, $\omega_{b}=2 \pi f_{b}, f_{b}$ is the frequency in $\mathrm{Hz}$ and is assumed to be small so that the wave effect can be neglected, $\phi$ is the lead angle in with respect to the heart action. Since the blood flow is assumed to be pulsatile, so the pressure gradient can be given as:

$-\frac{\partial \bar{p}}{\partial z}(z, t)=\overline{A_{0}}+\overline{\mathrm{A}_{1}} \cos \left(\overline{\omega_{p} t}\right)$,

where $\overline{A_{0}}$ is the steady component of the pressure gradient, $\overline{A_{1}}$ is the amplitude of the pulsatile component of the pressure gradient, $\overline{\omega_{p}}=2 \pi \overline{f_{p}}, \overline{f_{p}}$ is the frequency in $\mathrm{Hz}$. The constitutive equation for the Herschel-Bulkley fluid is given by,

For $\overline{\tau_{H B}} \geq \bar{\tau}_{y}$

$$
\overline{\tau_{H B}}=\overline{\mu_{H B}}\left(-\frac{\partial \bar{u}_{H B}}{\partial r}(z, t)\right)^{1 / n}+\bar{\tau}_{y},
$$

For $\overline{\tau_{H B}} \leq \bar{\tau}_{y} \frac{\partial \bar{u}_{H B}}{\partial \bar{r}}(z, t)=0$,

where $\bar{\tau}_{y}$ is the yield stress is of the H-B fluid and $\overline{\mu_{H B}}$ is the coefficient of viscosity of Herschel -Bulkley fluid. The geometry of the asymmetric shape of stenosis in the arterial segment is mathematically represented by;

$$
\begin{aligned}
& h(\bar{z})=d(\bar{z}) \\
& \left\{\begin{array}{c}
1-\bar{G}\left(b^{m-1}(\bar{z}-\bar{a})-(\bar{z}-\bar{a})^{m}\right), a \leq z \leq a+b \\
1, \text { otherwise. }
\end{array}\right.
\end{aligned}
$$

where $\bar{G}=\left(\frac{\bar{\delta}}{\overline{R_{0} L_{0}}}\right) m^{m /(m-1)}, d(z)=d_{0}+$ $\varepsilon Z$ and $\bar{\delta}$ denotes the maximum height of the stenosis at

$$
z=\bar{d}+\frac{\overline{L_{0}}}{m^{m /(m-1)}},
$$

such that $\frac{\bar{\delta}}{\bar{R}_{0}} \ll 1, L_{0}$ is the length of stenosis, $\mathrm{d}$ denotes its location, $\bar{R}(\mathrm{z})$ is the radius of the artery in the stenosed free region, $R_{0}$ is the radius of artery in the stenosed region, $\mathrm{m}$ is a parameter determining the shape constriction profile and referred to as a shape parameter. The boundary condition for the momentum and constitutive equations for the velocity and shear stress:

At $\bar{r}=0, \overline{\tau_{H B}}$ is finite

At $\bar{r}=R(z), \overline{u_{H B}}=0$.

Introducing the non-dimensional variables as:

$z=\frac{\bar{z}}{R_{0}}, R(z)=\frac{\overline{R(z)}}{R_{0}}, r=\frac{\bar{r}}{R_{0}}, t=\bar{\omega} \bar{t}, \omega=\frac{\overline{\omega_{b}}}{\overline{\omega_{p}}}$,

$\delta=\frac{\bar{\delta}}{R_{0}}, u_{H B}=\frac{4 \mu_{0} \overline{u_{H}}}{A_{0} R_{0}^{2}}, \overline{\tau_{H B}}=\frac{4 \mu_{0} \overline{\tau_{H B}}}{A_{0} R_{0}}, \theta=2 \frac{\bar{\tau}_{y}}{A_{0 R_{0}}}$

, ${\overline{\alpha_{H B}}}^{2}=\frac{\omega R_{0}^{2} \overline{\rho_{H B}}}{\overline{\mu_{H B}}}, e=\frac{\overline{A_{1}}}{\overline{\overline{A_{0}}}}, B=\frac{\overline{a_{0}}}{\overline{A_{0}}}, H=\frac{\bar{H}}{H_{0}},(9)$

where $\alpha_{H B}$ is the Womersely frequency parameter or pulsatile Reynolds number of Herschel-Bulkley fluid model. Substituting the non-dimensional parameters (9) in the momentum equation (1) and constitutive equation (3) we can have the following equations:

${\overline{\alpha_{H B}}}^{2} \frac{\partial u_{H B}}{\partial t}=4(1+e \cos t)+4 B \cos (\omega t+$ $\phi)-\frac{2}{r} \frac{\partial}{\partial r}\left(r \overline{\tau_{H B}}\right)+4 F_{1} \frac{\partial H}{\partial z} \cos \psi-\frac{R e}{F r} \sin \alpha$,

where $F_{1}=\frac{4 M H_{0} \mu_{0}}{\rho A_{0} R_{0}{ }^{2}}$, Reynolds number $R e=$ $\frac{\rho_{c} R_{0} u_{0}}{\mu_{H B}}$ and Froude number $F r=\frac{u_{0}^{2}}{g R_{0}}$.

For $\overline{\tau_{H B}} \geq \theta$

$$
\begin{aligned}
& \bar{\tau}_{H B}=\left(-\frac{1}{2} \frac{\partial \bar{u}_{H B}}{\partial r}(z, t)\right)^{1 / n}+\theta, \\
& \text { For } \overline{\tau_{H B}} \leq \theta \\
& \frac{\partial \bar{u}_{H B}}{\partial \bar{r}}(z, t)=0,
\end{aligned}
$$

Non-dimensional form for the boundary conditions can be re-written as:

At $=0, \overline{\tau_{H B}}$ is finite,

At $r=R(z), \overline{u_{H B}}=0$ 
The volumetric flow rate can be given by,

$Q=4 \int_{0}^{R(z)} r u_{H B} d r$

where $Q=\frac{8 \mu_{H B} \bar{Q}}{\pi \bar{A}_{0} R_{0}}, \bar{Q}$ is the volumetric flow rate. The resistive impedance for the flow can be written as:

$\lambda=\frac{\Delta p}{Q}=\frac{1}{Q}\left[\int_{0}^{a} \Delta p d z+\int_{a}^{a+b} \Delta p d z+\right.$

$\left.\int_{a+b}^{L_{0}} \Delta p d z\right]$,

\section{Solution of Equation}

The perturbation method is applied to solve the system of non-linear partial differential equations. Let us expand the velocity in the perturbation series about the square of the pulsatile Reynolds number as below (where \left.${\overline{\alpha_{H B}}}^{2} \ll 1\right)$

$$
\begin{aligned}
& \bar{u}_{H B}(r, z, t)=\bar{u}_{H B 0}(r, z, t)+ \\
& \frac{\alpha_{H B}}{\alpha_{H B 1}} \bar{u}_{H, z, t)+\cdots \ldots \ldots \ldots \ldots \ldots}(r, \ldots \ldots \ldots \ldots
\end{aligned}
$$

Similarly, one can expand the shear stress $\overline{\tau_{H B}}(r, z, t)$, the plug core radius $R_{p}(z, t)$, the plug core velocity $u_{p}(z, t)$, and the plug core shear stress $\overline{\tau_{p}}(z, t)$, in terms of ${\overline{\alpha_{c}}}^{2}$. Substituting the perturbation series expansions of $u_{H B}$ and $\tau_{H B}$ in [9] and then equating the constant term and ${\overline{\alpha_{H B}}}^{2}$ terms respectively one can obtain the first approximation and the second approximation as,

$\frac{\partial\left(r \tau_{H B}\right)}{\partial r}=2(1+e \cos t)+2 B \cos (\omega t+\phi)+$ $F_{1} \frac{\partial H}{\partial z} \cos \psi-\frac{R e}{F r} \sin \alpha$,

$\frac{\partial\left(u_{H B 0}\right)}{\partial t}=-\frac{2 \partial\left(r \tau_{H B 1}\right)}{r \partial r}$,

Substituting the perturbation series expansions of and in (10) and then equating the constant term and terms respectively one can obtain first approximation and the second approximation as,

$-\frac{\partial\left(u_{H B 0}\right)}{\partial r}=2 \tau_{H B 0}{ }^{n-1}\left[\tau_{H B 0}-n \theta\right]$,
$-\frac{\partial\left(u_{H B 1}\right)}{\partial r}=2 n \tau_{H B 0}{ }^{n-2} \tau_{H B 1}\left[\tau_{H B 0}-(n-\right.$

1) $\theta]$,

Applying the perturbation series expansion of $u_{H B}$ and $\tau_{H B}$ in the boundary conditions and then equating the constant terms and ${\overline{\alpha_{H B}}}^{2}$ term, we get:

$$
\tau_{H B 0}, \tau_{H B 1} \text { are finite at } r=0 .
$$$$
u_{H B 0}=0, u_{H B 1}=0 \text { at } r=0 \text {. }
$$

Solving (18) and (19) with the help of (20) we can have a solution as,

$$
\begin{aligned}
& \tau_{p 0}=g(t) R_{0 p}, \\
& \tau_{H B 0}=g(t) r, \\
& u_{H B 0}=2[g(t) R]^{n} R\left[\frac{1}{n+1}\left\{1-\left(\frac{r}{R}\right)^{n+1}\right\}-\right. \\
& \left.\frac{q^{2}}{R}\left\{1-\left(\frac{r}{R}\right)^{n}\right\}\right], \\
& u_{p 0}=2[g(t) R]^{n} R\left[\frac{1}{n+1}\left\{1-\left(\frac{q^{2}}{R}\right)^{n+1}\right\}-\right. \\
& \left.\frac{q^{2}}{R}\left\{1-\left(\frac{q^{2}}{R}\right)^{n}\right\}\right], \\
& \tau_{p 1}=-[g(t) R]^{n} D R^{2}\left[\frac{n}{2(n+1)}\left(\frac{q^{2}}{R}\right)-\right. \\
& \left.\frac{n-1}{2}\left(\frac{q^{2}}{R}\right)^{2}-\frac{n}{2(n+1)}\left\{\left(\frac{q^{2}}{R}\right)^{n+2}\right\}\right],
\end{aligned}
$$

$\tau_{H B 1}=-[g(t) R]^{n} D R^{2}\left[\frac{n}{(n+1)(n+3)}\left\{\frac{n+3}{2}\left(\frac{r}{R}\right)-\right.\right.$ $\left.\left(\frac{r}{R}\right)^{n+2}\right\}-\frac{n-1}{n+2}\left(\frac{q^{2}}{R}\right)\left\{\frac{n+2}{2}\left(\frac{r}{R}\right)-\left(\frac{r}{R}\right)^{n+1}\right\}-$ $\left.\frac{3 n\left(n^{2}+2 n-3\right)}{2(n+1)(n+3)}\left(\frac{R}{r}\right)\left(\frac{q^{2}}{R}\right)^{n+3}\right]$,

$u_{H B 1}=$

$-2 n[g(t) R]^{2 n-1} D R^{3}\left[\frac{n}{2(n+1)^{2}(n+3)}\{(n+\right.$ $\left.2)-(n+3)\left(\frac{r}{R}\right)^{n+1}+\left(\frac{r}{R}\right)^{2 n+2}\right\}+$ $\frac{n}{(n+1)(n+2)(n+3)(2 n+1)}\left(\frac{q^{2}}{R}\right) \times\{(n+2)(n+$ 3) $(2 n+1)\left[\left(\frac{r}{R}\right)^{n}+\left(\frac{r}{R}\right)^{n+1}\right]-2\left[\left(2 n^{3}+\right.\right.$ $\left.9 n^{2}+11 n+3\right)+\left(2 n^{2}+6 n+\right.$ 3) $\left.\left.\left(\frac{r}{R}\right)^{2 n+1}\right]\right\}+\frac{(n-1)^{2}}{2 n(n+2)}\left(\frac{q^{2}}{R}\right)^{2}\{(n+1)-$ $\left.(n+2)\left(\frac{r}{R}\right)^{n}+\left(\frac{r}{R}\right)^{2 n}\right\}+$ 
$\frac{3 n\left(n^{2}+2 n-2\right)}{2(n+1)(n+2)(n+3)}\left\{\left(\frac{r}{R}\right)^{n-1}-1\right\}\left(\frac{q^{2}}{R}\right)^{n+3}+$
$\left.\frac{3(n-1)\left(n^{2}+2 n-2\right)}{2(n+1)(n+2)(n+3)}\left\{1-\left(\frac{r}{R}\right)^{n-2}\right\}\left(\frac{q^{2}}{R}\right)^{n+4}\right]$,

$u_{p 1}=$

$-2 n[g(t) h]^{2 n-1} D h^{3}\left[\frac{n}{2(n+1)^{2}(n+3)}\{(n+\right.$

$\left.2)-(n+3)\left(\frac{q^{2}}{h}\right)^{n+1}+\left(\frac{q^{2}}{h}\right)^{2 n+2}\right\}+$

$\frac{(n-1)}{2(n+1)(n+2)(n+3)(2 n+1)}\left(\frac{q^{2}}{h}\right) \times\{(n+2)(n+$

3) $(2 n+1)\left[\left(\frac{q^{2}}{h}\right)^{n}+\left(\frac{q^{2}}{h}\right)^{n+1}\right]-$

$2\left[\left(2 n^{3}+9 n^{2}+11 n+3\right)+\left(2 n^{2}+6 n+\right.\right.$

3) $\left.\left.\left(\frac{q^{2}}{h}\right)^{2 n+1}\right]\right\}+\frac{(n-1)^{2}}{2 n(n+2)}\left(\frac{q^{2}}{h}\right)^{2}\{(n+1)-$

$\left.(n+2)\left(\frac{q^{2}}{h}\right)^{n}+\left(\frac{q^{2}}{h}\right)^{2 n}\right\}+$

$\frac{3 n\left(n^{2}+2 n-2\right)}{2(n+1)(n+2)(n+3)}\left\{\left(\frac{q^{2}}{h}\right)^{n-1}-1\right\}\left(\frac{q^{2}}{h}\right)^{n+3}+$

$\left.\frac{3(n-1)\left(n^{2}+2 n-2\right)}{2(n+1)(n+2)(n+3)}\left\{1-\left(\frac{q^{2}}{h}\right)^{n-2}\right\}\left(\frac{q^{2}}{h}\right)^{n+4}\right]$,

where $q^{2}=\frac{\theta}{g(t)}, g(t)=[2[1+e \cos t]+$

$B \cos (\omega t+\emptyset)]+F_{1} \frac{\partial H}{\partial z} \cos \psi-\frac{R e}{F r} \sin \alpha$ and

$D=\frac{1}{g} \frac{d g}{d t}$.

The expression for wall shear stress

$$
\tau_{w}=\left(\tau_{c 0}+\alpha_{c}{ }^{2} \tau_{c 1}\right)_{r=R} .
$$

The expression for the volumetric flow rate

$$
\begin{aligned}
Q(z, t)=4[ & {\left[\int_{0}^{R_{p 0}} r u_{p 0} d r+\int_{R_{p 0}}^{R} r u_{p 0} d r\right) } \\
& +\alpha_{c}^{2}\left(\int_{0}^{R_{p 0}} r u_{p 0} d r\right. \\
& \left.\left.+\int_{R_{p 0}}^{R} r u_{p 0} d r\right)\right]
\end{aligned}
$$

The expression for the plug core radius can be written as:

$$
\begin{array}{r}
R_{p}=q^{2}+\alpha^{2}[g(t) h]^{n-1} \frac{n D h^{3}}{2(n+1)}\left[\left(\frac{q^{2}}{R}\right)\right. \\
\left.-\frac{n^{2}-1}{n}\left(\frac{q^{2}}{R}\right)^{2}-\left(\frac{q^{2}}{R}\right)^{n+2}\right]
\end{array}
$$

The longitudinal impedance to flow in the artery is defined as: $\lambda=\frac{P(t)}{Q(z, t)}$, where $p(t)=$ $4(1+e \cos t)$ is a pressure gradient in the non-dimensional form.

\section{Result and Discussion}

In converging and diverging region, for the analysis of the salient features of the Herschel Bulkley blood flow model, the effect of vital parameters defining flow geometries and fluid behavior such as Reynolds Number, Froude number, inclination angle for artery $(\alpha)$ as well as of magnetic field $(\theta)$ on the flow characteristic plug flow radius, wall shear stress, shear stress at stenosis throat, axial velocity and volumetric flow rate are discussed numerically with computational illustrations. All graphs are plotted for the values of $\sigma=$ $\frac{b}{a}=0, b=1, \xi=\tan \phi=0.005,0,-0.005$, $R e=0.1, F r=0.1, M=2,3,4$ and shape parameter $m=2,6$ and 11 with the stenosis height from 0 to 0.2 using mathematica software. Fig. 2 represents the variation of volumetric flow rate with the pressure gradient and yield stress. This graph shows that with the increase of yield stress, the volumetric flow rate increases. This could be because of the decrease in plug flow region with the increase of yield stress. With the increase of pressure gradient, the flow rate increases confirming that the plug flow region is reduced. Fig. 3 represents the variation of impedance with an axial distance. The resistive impedance in a diverging region is smaller than those in converging region because of the flow rate is higher in the former than those in latter, as anticipated. Obstruction to the flow is less in case of diverging artery as compared to the converging artery. Fig. 4 represents that impedance appears to be uniform for smaller values of pressure gradient for both the cases of yield stress. It appears from the graph that the impedance is more for the converging region as compared to diverging region. Fig. 5 represents that volumetric flow rate appears to maximum in core region of the artery. For the converging region the flow rate is less as compared to the diverging region. 


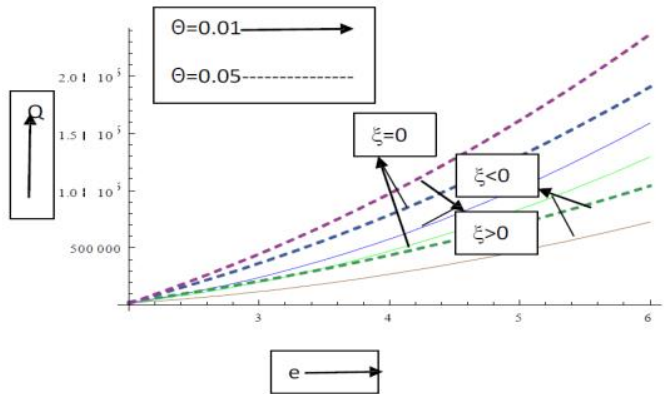

Fig. 2 Variation of volumetric flow rate with a pressure gradient ' $\mathrm{e}$ '.

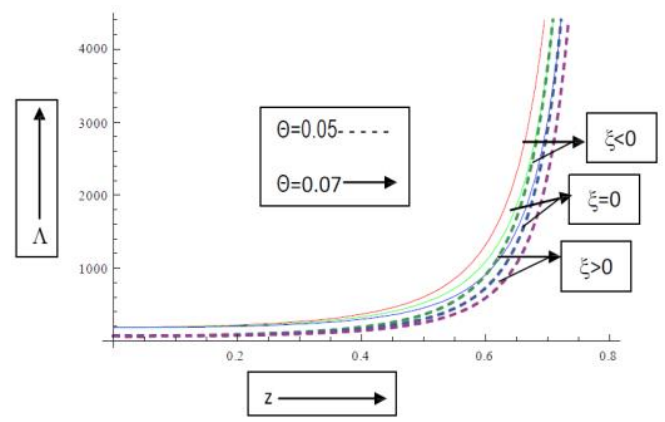

Fig. 3 Variation of impedance 'A' with an axial distance ' $z$ '.

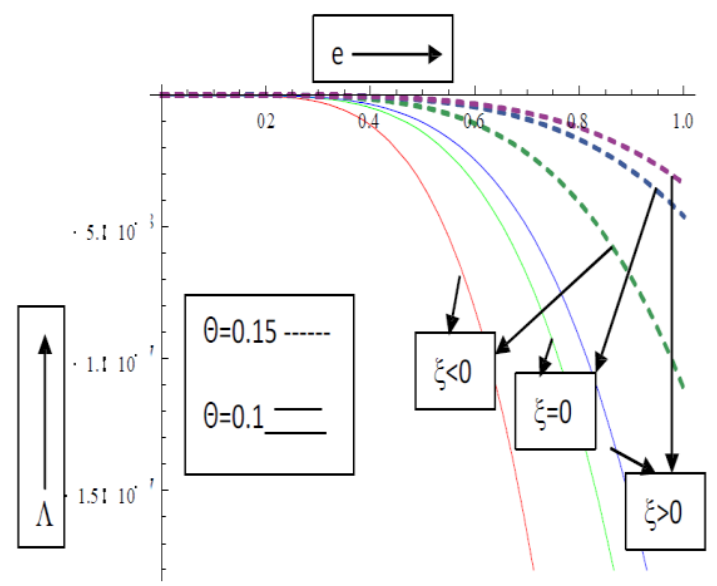

Fig. 4 Variation of impedance ' $A$ ' with pressure gradient 'e'.

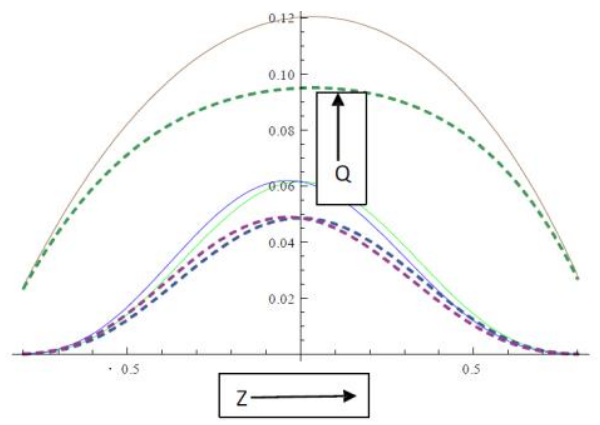

Fig. 5 Variation of volumetric flow rate with the axial distance ' $\mathrm{z}$ '.

\section{Conclusions}

The present study deals with the analysis of flow characteristics of the blood flowing through an inclined stenosed tapered artery under the influence of an inclined magnetic field. This investigation can play a vital role in the determination of axial velocity, volumetric flow rate and impedance in particular situations and some interesting predictions related to the medical interest has been analysed. The main findings of the present mathematical analysis are as follows; with the increase of pressure gradient, the flow rate increases confirming that the plug flow region is reduced; obstruction to the flow is less in case of accelerated diverging artery as compared to the accelerated converging artery; volumetric flow rate appears to be maximum in the core region of the artery.

\section{Acknowledgement}

The author is thankful to the college authorities for providing the necessary help.

\section{References}

1. Abdullah I. and Amin N., (2010) A micropolar fluid model of blood flow through a tapered artery through a stenosis, Math. Metn. Appl. Sci., Vol. 33, pp.1910-1923.

2. Neetu Srivastava (2014) Analysis of Flow Characteristics of the Blood Flowing through an Inclined Tapered Porous Artery with Mild Stenosis under the Influence of an Inclined Magnetic Field, Journal of Biophysics, Vol. 2014, Article ID 797142, 9 pages.

3. Neetu Srivastava (2014) The Casson fluid model for blood flow through an inclined tapered artery of an accelerated body in the presence of magnetic field, Int. J. Biomedical Engineering and Technology, Vol. 15, No. 3.

4. Abdullah I., Amin N. and Hayat T., (2011) Magnetohydrodynamic effects on blood flow through an irregular stenosis, Int. J. Num. Meth. Fluid. Vol. 67, pp. 1624-1636.

5. Bodnar T., Sequeira A., and Prosi M., (2011) On the shear-thinning and viscoelastic effects of blood flow 
under various flow rates, Appl. Math. Comput. Vol. 217, pp. 5055-5056.

6. Mekheimer Kh. S. and Elkot M. A. (2012) Mathematical modeling of unsteady flow of a Sisko fluid through an anisotropically tapered elastic arteries with timevariant overlapping stenosis, Appl. Math. Model. Vol. 36, pp. 5393-5407.

7. Ali N., Zaman A., and Sajid M., (2014) Unsteady blood flow through a tapered stenotic artery using Sisko model, Computers and Fluids, Vol. 101, pp. 42-49.

8. Akbar N.S. and Nadeem S., (2014) Carreau fluid model for blood flow through a tapered artery with a stenosis, A. Shams Eng. J. 5, pp. 13071316.

9. Priyadharshini S. and Ponalagusamy R. (2015) Biorheological Model on Flow of Herschel-Bulkley Fluid through a Tapered Arterial Stenosis with Dilatation, Applied Bionics and Biomechanics, Vol. 2015. Article ID 406195, 12 pages.

10. Somchai Sriyab (2014) Mathematical analysis of Non-Newtonian Blood Flow in Stenosis Narrow Arteries, Computational and Mathematical Methods in Medicine, Vol.2014, Article ID 479152, 10 pages.

11. Singh A. K. (2013) MHD Flow of Blood through Radially Nonsymmetric Stenosed Artery: a Hershcel-Bulkley Model, International Journal of Engineering, Transactions B: Applications, Vol. 26, 8 (B). 